PROFESIONALES Y HERRAMIENTAS PARA EL DESARROLLO LOCAL Y SUS SINERGIAS TERRITORIALES. EVALUACIÓN Y PROPUESTAS DE FUTURO IX Coloquio Nacional de Desarrollo Local del GTDL-AGE 

ANTONIO MARTÍNEZ PUCHE, XAVIER AMAT MONTESINOS, ISABEL SANCHO CARBONELL y DANIEL SANCHIZ CASTAÑO (EDS.)

\section{PROFESIONALES Y HERRAMIENTAS PARA EL DESARROLLO LOCAL Y SUS SINERGIAS TERRITORIALES. EVALUACIÓN Y PROPUESTAS DE FUTURO}

IX Coloquio Nacional de Desarrollo Local del GTDL-AGE

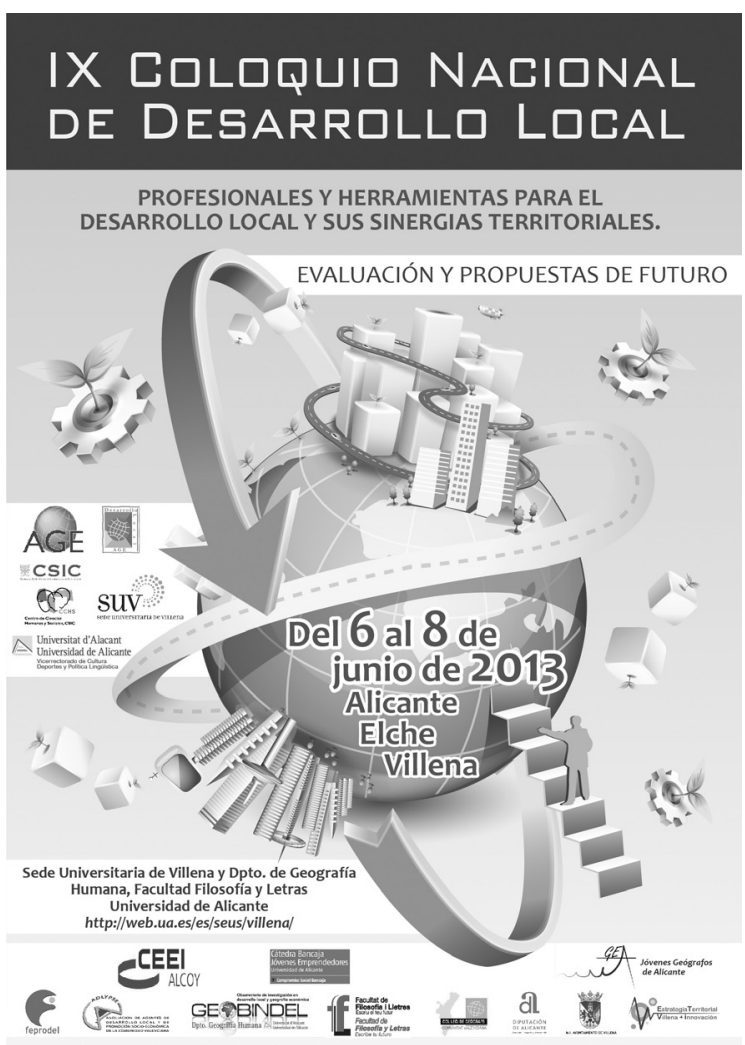


Este libro ha sido examinado y valorado por evaluadores ajenos a la Universidad de Alicante, con el fin de garantizar la calidad científica del mismo.

Publicacions de la Universitat d'Alacant

03690 Sant Vicent del Raspeig

Publicaciones@ua.es

http://publicaciones.ua.es

Telèfon: 965903480

(C) Antonio Martínez Puche, Xavier Amat Montesinos,

Isabel Sancho Carbonell y Daniel Sanchiz Castaño (eds.), 2016

(C) d'aquesta edició: Universitat d'Alacant

ISBN: 978-84-16724-00-0

Dipòsit legal: A 92-2016

Disseny de coberta: candela ink

Composició: Página Maestra (Miguel Ángel Sánchez Hernández)

Impressió i enquadernació: Guada Impresores

\section{unte \\ Unión de Editoriales
Universitarias Españolas \\ WWW.une.es
WWA}

Esta editorial es miembro de la UNE, cosa que garantiza la difusión y comercialización nacional y internacional de sus publicaciones.

Reservados todos los derechos. Cualquier forma de reproducción, distribución, comunicación pública o transformación de esta obra sólo puede ser realizada con la autorización de sus titulares, salvo excepción prevista por la ley. Diríjase a CEDRO (Centro Español de Derechos Repográficos, www.cedro.org) si necesita fotocopias o escanear algún fragmento de esta obra. 


\title{
PROFESIONALES EN DESARROLLO LOCAL. ACCIONES, LOGROS Y RETOS DE FUTURO ¿QUÉ VA A PASAR CON LOS AEDL?
}

\author{
Javier González Cardona \\ Presidente de la Federación Española de Profesionales de Desarrollo Local (FEPRODEL) \\ javigoncar@gmail.com
}

\section{RESUMEN}

Desde que en febrero de 2013 se hizo público el informe sobre el anteproyecto de Ley de racionalización y sostenibilidad de la Administración Local, se ha generado una situación de incertidumbre, no solamente entre los técnicos en gestión del desarrollo local, sino también entre la mayoría de alcaldes, concejales y profesionales que trabajan en las diferentes Administraciones locales. Una de las primeras entidades en pronunciarse contrariamente al respecto ha sido FEPRODEL, llevando a cabo acciones informativas y trasladando sus argumentos tanto al Ministerio de Hacienda y Administraciones Públicas como a la Federación Española de Municipios y Provincias.

Esto ocurre en un contexto donde, curiosamente, el desarrollo local vuelve a poner de manifiesto su capacidad de dar respuesta a situaciones difíciles y de generar entornos favorables para la creación de actividad económica, empleo y cohesión social. Así lo pone de manifiesto el reciente Dictamen del Comité de las Regiones de la Unión Europea sobre el Desarrollo Local Participativo. El avance hacia un modelo socioeconómico de estas características no sería posible sin la importante red de profesionales técnicos en gestión del desarrollo local existente en España. Un colectivo cada vez más amplio que apuesta por la creación de un Colegio Profesional que refuerce su aportación a la sociedad.

Palabra clave: Desarrollo Local, FEPRODEL, Colegio Profesional, ciudadanía

PROFESSIONALS IN LOCAL DEVELOPMENT. ACTIVITIES, ACHIEVEMENTS AND FUTURE CHALLENGES WHAT WILL HAPPEN WITH AEDL? 


\section{ABSTRACT:}

Since February, 2013, was made public the report on the draft Law on rationalization and sustainability of local government, has created a situation of uncertainty, not only among technicians in local development management, but also among the most mayors, councilors and professionals working in different local authorities. One of the first entities to rule contrary in this regard has been FEPRODEL, conducting information campaigns and translating their arguments both the Ministry of Finance and Public Administration as the Spanish Federation of Municipalities and Provinces.

This happens in a context where, interestingly, the local development again highlighting its ability to respond to difficult situations and to create environments conducive to the creation of economic activity, employment and social cohesion. This was evidenced by the recent opinion of the Committee of the Regions of the European Union on Participatory Local Development. Progress toward a socioeconomic model of this kind would not be possible without the extensive network of technical professionals in existing local development management in Spain. A widening group that pursues the creation of an official professional association to improve its contribution to society.

Key words: Local Development, FEPRODEL, Professional Association, citizenship

\section{LAS COMPETENCIAS DE LOS AYUNTAMIENTOS EN DESARROLLO LOCAL}

Aunque la definición de las competencias de los ayuntamientos españoles nunca ha estado bien resuelta, el anteproyecto Ley de racionalización y sostenibilidad de la Administración Local anunciado recientemente persigue, entre otros objetivos, clarificar las competencias municipales y racionalizar la estructura de la Administración Local, de acuerdo con los principios de estabilidad, sostenibilidad y eficiencia.

Sin embargo, una vez analizado el contenido del texto, se observa que en ningún momento se hace referencia al empleo, y al fomento de la actividad emprendedora como competencias municipales propias, ni siquiera como competencias susceptibles de ser delegadas por otras Administraciones a la Administración Local.

Tampoco se hace ninguna referencia a la importancia del desarrollo local, entendido como un proceso activador de la economía y dinamizador de la sociedad local que a través del aprovechamiento eficiente y sostenible de los recursos existentes en el territorio, favorece la creación de nuevas actividades económicas y empresas, genera empleo y contribuye a mejorar la calidad de vida de la población local. 
El texto del Anteproyecto está olvidando de este modo incluir una realidad fácilmente constatable en los entornos locales más cercanos a las personas. Y es que la importancia de la dimensión local de las políticas de empleo ha sido ampliamente reconocida por la ciudadanía, los agentes económicos y sociales, partidos políticos y las diferentes Administraciones Públicas durante las tres últimas décadas.

La importancia del empleo viene dada porque es la base de nuestro sistema socioeconómico, ya que proporciona ingresos a la ciudadanía, confiere libertad individual, asegura progreso económico, garantiza cohesión y solidaridad social y ofrece seguridad material. De ahí, la imperiosa necesidad de situar el trabajo en el centro de las políticas diseñadas por los agentes públicos y de las decisiones económicas adoptadas por los agentes privados. La creación de más y mejores empleos ha de ser un objetivo irrenunciable y compartido por todas las Administraciones Públicas. No podemos olvidar que en un mundo global el empleo continúa generándose a escala local.

Sin ir más lejos, en marzo del 2010 la UE aprobó la Estrategia Europa 2020, eje central de las políticas comunitarias para los próximos años. Con esta estrategia se pretende dirigir el desarrollo de la UE hacia un crecimiento inteligente, sostenible e integrador, fomentando una economía con alto nivel de empleo que tenga cohesión social y territorial. Para conseguir estos objetivos, la Estrategia establece tareas a todos niveles administrativos, indicando que las autoridades nacionales, regionales y locales deberían colaborar entre sí, y asociarse con otros agentes sociales para elaborar los programas de reforma y su aplicación.

En el mismo sentido, el Dictamen del Comité de las Regiones de 29 de noviembre de 2012 sobre Desarrollo Local Participativo, considera que, de cara al próximo período de programación comunitaria, se necesita más apoyo de los fondos europeos a las políticas de desarrollo local, para posibilitar no solo que los ciudadanos perciban en mayor medida como propios los objetivos de la Estrategia Europa 2020, sino también para facilitar e impulsar la recuperación económica y la creación de empleo.

Por otro lado, la Estrategia Española de Empleo y la vigente Ley 56/2003, de 16 de diciembre, de Empleo, reflejan la importancia de la dimensión local en este ámbito y establecen que las políticas de empleo, en su diseño y modelo de gestión, deberán tener en cuenta su dimensión local para ajustarlas a las necesidades del territorio, de manera que favorezcan y apoyen las iniciativas de generación de empleo en el ámbito local.

Desde un punto de vista político, en las últimas elecciones municipales españolas de 2011, todos los partidos con mayor representación en las Corporaciones Locales incluyeron el empleo y la creación de empresas como dos de las principales propuestas en sus respectivos programas electorales municipales. 
En consecuencia, la Administración Local, la mejor valorada por los ciudadanos según el CIS, ha venido prestando servicios relacionados con el empleo y la creación de empresas, principalmente por tres motivos. En primer lugar, por la propia demanda y necesidades de la ciudadanía. En segundo lugar, por los incentivos que en forma de subvenciones procedentes de otras Administraciones Públicas se han dado para estos fines. Y en tercer lugar, por la aparición constante de normativa, en ámbitos como las políticas activas de empleo, el comercio, el turismo o el desarrollo rural sostenible, donde se ha contemplado a la Administración Local como un actor clave en el fomento de empleo y de la actividad emprendedora local. Ello ha provocado la aparición paulatina y su posterior consolidación de servicios municipales de empleo y fomento de la actividad emprendedora en todo el territorio estatal.

Estos servicios municipales de creación de empleo y fomento de la actividad emprendedora, denominados en la mayoría de los casos Agencias de Desarrollo Local, se han convertido en el principal servicio de apoyo a los emprendedores y a la creación de empleo en España y han venido contando con el reconocimiento y colaboración del Estado, las Comunidades Autónomas a través de los Servicios Autonómicos de Empleo, la Unión Europea y otros organismos internacionales como la OCDE. También han contado con el reconocimiento de las universidades, las empresas y diferentes agentes económicos y sociales.

Y por supuesto, los ayuntamientos también han marcado su postura en este ámbito. El informe de la Federación Española de Municipios y Provincias (FEMP) "Gasto local en políticas activas de empleo", del año 2011, indica que las políticas activas de empleo cada vez tienen más presencia en los ayuntamientos. De esta manera el $83 \%$ de los municipios de más de 500 habitantes cuentan con personal y servicios de empleo y el $90 \%$ realizó algún gasto en este campo durante 2009. Esto viene a demostrar que la mayoría de los ayuntamientos ha considerado que era necesario contar con servicios dedicados al desarrollo socioeconómico local.

La propia FEMP incide en este hecho cuando, en su asamblea general de 2011, aprueba un conjunto importante de resoluciones relacionadas con las demandas en la mejora de los servicios y en la aplicación de las políticas de empleo. Y además dedica toda una ponencia a la economía y desarrollo local, incorporando una comunicación específica sobre políticas activas de empleo y nueva economía, donde se indica que frente a la crisis económica los Gobiernos Locales deben ser los grandes aliados del cambio de modelo económico y del impulso a las políticas activas de empleo junto a otras Administraciones Públicas.

Recientemente, la FEMP también ha presentado alegaciones al anteproyecto de ley, solicitando que se incluya el desarrollo económico local como competencia municipal. 


\section{El DESARrollo local PARTicipativo}

Tal como se ha citado en el apartado anterior, recientemente, el Comité de las Regiones de la Unión Europea emitió un Dictamen sobre el desarrollo local participativo indicando que los elementos de valor añadido de este tipo de desarrollo son la participación, la concertación y cooperación de la población local y de todos los agentes públicos y privados locales, la adecuación de la estrategia de desarrollo local a las necesidades específicas de cada territorio, una poderosa influencia en la colaboración multinivel e intersectorial, la movilización de unos sólidos conocimientos técnicos locales, la capacidad de innovación a nivel local, el enfoque integrado y multisectorial, unas acciones y resultados establecidos en el ámbito local, y un planteamiento flexible y estratégico.

En estos difíciles momentos de crisis y recesión económica, la cooperación entre las diferentes Administraciones Públicas es más oportuna que nunca para permitir la aplicación de políticas de empleo tanto en las comarcas urbanas como en las rurales, teniendo mejor en cuenta y centrándose en las necesidades específicas de las personas y de cada territorio.

La perspectiva territorial, contemplada también en la Estrategia Española de Empleo, implica que las Políticas Activas de Empleo deben adaptarse a las características y necesidades específicas que se derivan de los diferentes niveles de organización territorial del Estado, impulsando y promoviendo el desarrollo en todos los tipos de territorio en los que este se configura. El enfoque local es un marco idóneo para la detección y el aprovechamiento de nuevas posibilidades productivas o explotadas todavía, por lo que resulta imprescindible impulsar y gestionar en él actuaciones que tengan por objeto la creación de empleo y la actividad empresarial.

3. LA NECESIDAD DE UN COLEGIO OFICIAL COMO REFERENTE DEL COLECTIVO PROFESIONAL

En las últimas fechas, desde FEPRODEL se está avanzando firmemente hacia la creación del Colegio Profesional de Técnicos en Gestión del Desarrollo Local. Tras alcanzar un consenso con las diferentes asociaciones autonómicas respecto al nombre y las características del futuro Colegio, se ha iniciado una campaña de apoyos individuales e institucionales que pretende dar sus frutos en los próximos meses.

Un Colegio Profesional es una Corporación de derecho público, amparada por la Ley y reconocida por el Estado y las Comunidades Autónomas, con personalidad jurídica propia y plena capacidad para el cumplimiento de sus fines.

a) Un Colegio Profesional tiene como fines esenciales:

b) La ordenación del ejercicio de una profesión.

c) La representación institucional de la misma.

d) La defensa de los intereses profesionales de los colegiados. 
e) La protección de los intereses de los consumidores y usuarios de los servicios de sus colegiados.

La Constitución Española ampara la creación de Colegios Profesionales. En su artículo 36 establece: La ley regulará las peculiaridades propias del régimen jurídico de los Colegios Profesionales y el ejercicio de las profesiones tituladas. La estructura interna y el funcionamiento de los Colegios, deberán ser democráticos.

Por otra parte, tanto la Ley Estatal 2/1974, de 13 de febrero, sobre Colegios Profesionales como las Leyes Autonómicas se vieron afectadas por la Ley 25/2009, de 22 de diciembre, de modificación de diversas leyes para su adaptación a la Ley sobre el libre acceso a las actividades de servicios y su ejercicio). Esta última ley moderniza los Colegios, amplía sus fines esenciales, los abre a la competencia integrando más a los profesionales en la sociedad y facilita la colegiación, haciendo su gestión más transparente y protegiendo a los consumidores y usuarios.

\section{LA NECESIDAD SOCIAL DE LA PROFESIÓN}

Aunque el programa de Agentes de Empleo y Desarrollo Local se pone en marcha por primera vez en 1986, la implantación mayoritaria del mismo en los municipios españoles no se produce hasta mediados de la primera década del siglo actual. Para esta implantación, no exenta de trabas y dificultades, ha sido un elemento fundamental la subvención a la contratación de profesionales que las Corporaciones Locales han venido percibiendo durante todos estos años desde el Ministerio de Trabajo y los correspondientes Servicios de Empleo de las diferentes Comunidades Autónomas.

Como se ha señalado anteriormente, la referencia que la Ley de Empleo hace a la dimensión local de las políticas activas de empleo y a la necesaria coordinación que debería existir entre las Administraciones Públicas de distinto nivel para la gestión de las mismas, no se ha visto reflejada en una definición de competencias que enmarquen la dimensión territorial de las políticas de empleo.

En este estado de cosas, el desarrollo local y los profesionales que lo gestionan se han convertido en el principal referente de la participación de los ayuntamientos en las políticas activas de empleo. Esta realidad ha sido ampliamente reconocida por las diferentes Administraciones Públicas, Universidades y Agentes Económicos y Sociales, entre otros.

De este modo, el Ministerio de Trabajo, a través de la Orden TAS/360/2008', señalaba: Los Agentes de Empleo y Desarrollo Local se configuran como un

1 ORDEN TAS/360/2008, de 6 de febrero, por la que se modifica la Orden de 15 de julio de 1999, por la que se establecen las bases de concesión de subvenciones públicas para el fomento del desarrollo local e impulso de los proyectos y empresas calificadas como I + E. 
elemento clave en la dinamización de las políticas activas de empleo en el entorno local, impulsando y gestionando actuaciones que tienen por objeto la creación de empleo y la actividad empresarial. Los años de implantación de este programa han puesto de manifiesto por un lado la necesidad que las corporaciones locales tienen de contar con la figura del Agente de Empleo y Desarrollo Local y por otro, la oportunidad de aprovechar al máximo la experiencia de los técnicos cuyas contrataciones han sido objeto de subvención.

Actualmente en España existen más de 8.000 profesionales del desarrollo local que están contribuyendo directamente a la creación de una media anual de más de 40.000 empresas y a una inserción laboral que oscila entre las 320.000 y las 680.000 personas al año, estando consideradas las Agencias de Desarrollo Local como la principal plataforma de apoyo a emprendedores existente en nuestro país ${ }^{2}$.

Estos datos sitúan a las políticas de desarrollo local como una de las más eficientes y rentables para la inversión pública, debido a su enorme impacto y al efecto multiplicador que han generado en todos sus años de implantación.

Así pues, en lo referente la necesidad social de la profesión para poder proceder a la inscripción de un Colegio Profesional, ésta quedaría acreditada claramente por el papel clave que desempeñan Técnicos en Gestión del Desarrollo Local en las políticas activas de empleo.

\section{Perfil profesional. Funciones de los técnicos en Gestión del desarrollo local}

A pesar de lo señalado anteriormente en cuanto a la importancia del programa y de los profesionales que lo hacen posible, los avances referidos a la definición de funciones y del perfil profesional de los agentes de empleo y desarrollo local han sido muy escasas desde el punto de vista normativo. No ha ocurrido lo mismo en lo referente al asociacionismo profesional y a la oferta formativa universitaria.

Para la definición de las funciones profesionales se han escogido todas aquellas que vienen amparadas por la normativa estatal y autonómica.

La diferente normativa reguladora de las subvenciones ha incluido siempre un apartado dedicado a las funciones de los Agentes de Empleo y Desarrollo Local que ha variado bien poco. Estas funciones son las siguientes:

a. Colaborar en la promoción e implantación de las políticas activas de empleo relacionadas con la creación de actividad empresarial, desarrollándose dicha colaboración en el marco de actuación conjunta y acordada de la entidad contratante y los Servicios Públicos de Empleo (misión principal).

2 Elaboración de FEPRODEL a partir de datos y estadísticas del Ministerio de Empleo, la Federación Española de Municipios y Provincias, la Dirección General de Política de la PYME y los Servicios Autonómicos de Empleo, en el periodo 2007-2011. 
b. Prospección de recursos ociosos o infrautilizados, de proyectos empresariales de promoción económica local e iniciativas innovadoras para la generación de empleo en el ámbito local, identificando nuevas actividades económicas y posibles emprendedores.

c. Difusión y estímulo de potenciales oportunidades de creación de actividad entre los desempleados, promotores y emprendedores, así como instituciones colaboradoras.

d. Acompañamiento técnico en la iniciación de proyectos empresariales para su consolidación en empresas generadoras de nuevos empleos, asesorando e informando sobre la viabilidad técnica, económica y financiera y, en general, sobre los planes de lanzamiento de las empresas.

e. Apoyo a promotores de las empresas, una vez constituidas éstas, acompañando técnicamente a los mismos durante las primeras etapas de funcionamiento, mediante la aplicación de técnicas de consultoría en gestión empresarial y asistencia en los procesos formativos adecuados para coadyuvar a la buena marcha de las empresas creadas.

f. Cualesquiera otras que contribuyan a garantizar la misión principal.

Como acabamos de observar, si atendemos únicamente a la normativa estatal, las funciones de los agentes de empleo y desarrollo local estarían exclusivamente vinculadas a la creación de actividad empresarial. Sin embargo, la realidad nos muestra como las funciones de los profesionales del desarrollo local son mucho más amplias y variadas y así se ha venido reconociendo en los últimos años.

Por una parte, algunas Comunidades Autónomas han comenzado a incluir nuevas funciones para los AEDL, ampliándolas a otras políticas activas de empleo que van más allá de la creación de actividad empresarial.

En este sentido, la Comunidad Autónoma de Extremadura añade las siguientes funciones:

a. Comunicación, información y asesoramiento a las empresas y personas emprendedoras de su territorio acerca de las subvenciones destinadas al fomento del empleo, a la creación de iniciativas empresariales y a la mejora de la competitividad de las empresas existentes. Igualmente, facilitarán el acceso a dichas subvenciones prestando su apoyo en la gestión y tramitación de las mismas.

b. Colaboración en la difusión y utilización de aquellos servicios y desarrollos tecnológicos que el Servicio Extremeño Público de Empleo ponga a disposición de las personas usuarias del mismo para una mejor atención a éstas.

c. Apoyo al Servicio Extremeño Público de Empleo en la implementación de programas de orientación e información profesional, dirigidos a asesorar sobre las oportunidades de acceso al empleo y sobre las ofertas de formación, con el fin de facilitar la inserción y reinserción laboral 
de las personas que demandan empleo, en especial de las participantes en políticas activas de empleo. Asimismo, colaborarán en la captación de ofertas de empleo, identificando las necesidades de personal de las empresas a corto y medio plazo y proponiendo acciones de mejora de la empleabilidad de la población desempleada de su zona de actuación.

d. Colaboración con los Centros de Empleo del Servicio Extremeño Público de Empleo y con el resto de Agentes de Empleo y Desarrollo Local que trabajen en la Comunidad Autónoma de Extremadura en la mejora continua de la calidad de su servicio, intercambiando experiencias y conocimientos, así como desarrollando actividades y proyectos conjuntos.

e. Difusión de las medidas que se establezcan en los Planes de Empleo de la Junta de Extremadura, así como los específicamente elaborados por el Servicio Extremeño Público de Empleo.

f. Promoverán la participación e implicación de los agentes sociales y económicos, institucionales y de la ciudadanía en el desarrollo socioeconómico del territorio.

g. Fomento de la cultura emprendedora y del espíritu emprendedor.

h. Colaboración en el estudio del mercado de trabajo y el tejido empresarial de la comarca.

i. Diseñarán, elaborarán y ejecutarán proyectos de desarrollo local.

En Aragón, en el marco de colaboración con el Instituto Aragonés de Empleo para la promoción e implantación de las políticas activas de empleo, añaden las siguientes funciones a realizar por los agentes de empleo y desarrollo local:

a. Informarán y comunicarán a las empresas y emprendedores de su territorio acerca de las subvenciones para la formación y el empleo convocadas y vigentes, así como facilitarán el acceso a las mismas prestando su apoyo en la gestión y tramitación de las mismas.

b. Colaborarán en la difusión y utilización de aquellos servicios y desarrollos tecnológicos que el Instituto Aragonés de Empleo ponga a disposición de sus usuarios, -empresas y personas demandantes de empleo-, para una mejor atención a los mismos.

c. Facilitarán la inserción laboral de las personas demandantes de empleo, en especial de las participantes en políticas activas de empleo, identificando las necesidades de personal de las empresas o corto y medio plazo y proponiendo acciones de mejora de la empleabilidad de la población desempleada de su zona de actuación.

d. Analizarán la evolución y repercusión de las políticas activas de empleo en su zona de actuación, facilitándole la información requerida por el Instituto Aragonés de Empleo.

e. Colaborarán con el resto de Agentes de Empleo y Desarrollo Local que trabajen en la Comunidad Autónoma de Aragón en la mejora continua 
de la calidad de su servicio, intercambiando experiencias y conocimientos, así como desarrollando actividades y proyectos conjuntos.

En Castilla La Mancha se incorporan las siguientes funciones:

a. Apoyar a los empleadores del territorio en la búsqueda de trabajadores en colaboración con la oficina de empleo.

b. Actualizar la información que se les demanda desde la red/intranet, en relación con las actuaciones que llevan a cabo, dando la suficiente publicidad a los programas desarrollados por la Administración autonómica.

c. Suministrar la información que le requiera el Sepecam y prestar colaboración para la puesta en marcha de proyectos relacionados con la generación de empleo liderados por el mismo.

En el caso de Galicia se añaden las siguientes funciones:

a. Gestionar ayudas para favorecer el empleo, centrándose sobre todo, en aquellos colectivos con mayores dificultades a la hora de conseguir empleo.

b. Informar a las empresas de los instrumentos de promoción y fomento de la competitividad empresarial disponibles, así como facilitar su acceso a los mismos.

c. Promover la cultura emprendedora fomentando iniciativas empresariales y apoyando nuevos proyectos de autoempleo individual o colectivo informando de las medidas de apoyo existentes y poniendo en relación personas emprendedoras y recursos.

d. Comunicar a empresas y personas emprendedoras las subvenciones para la formación y el empleo, así como facilitar el acceso a las mismas.

e. Facilitar la inserción laboral de las personas demandantes de empleo, en especial de las participantes en políticas activas de empleo, identificando las necesidades de personal de las empresas a corto y medio plazo y proponiendo acciones de mejora de la empleabilidad de la población desempleada.

f. Observación estratégica del territorio y realización de estudios con el fin de detectar necesidades formativas y educativas de la población desempleada.

En el caso de la Comunitat Valenciana, se contemplan las siguientes funciones añadidas:

a. Acciones de difusión e información sobre los servicios y políticas activas del Servef, asesoramiento y apoyo sobre los programas de subvenciones en materia de formación, inserción y empleo.

b. Participación en jornadas, foros, eventos e intercambio de experiencias en relación con las políticas de empleo.

c. Detección de necesidades formativas o de cualquier otra acción que contribuya a garantizar la misión principal enunciada en el artículo anterior. 
d. Atender las instrucciones del Servef y cumplimentar los datos que le sean requeridos en la aplicación informática diseñada al efecto.

e. Establecer relaciones de coordinación con el Servef y con el Pacto Territorial de Empleo que pudiera existir en su territorio.

Aunque los servicios autonómicos de empleo han aprovechado esta ampliación de funciones para conseguir una mayor coordinación y ejercer más control sobre los AEDL, se confirma la presencia de estos profesionales en el ámbito de todas las, políticas activas de empleo, no solamente en aquellas que tienen relación con la creación de actividad empresarial sino también en aquellas otras relacionadas con la información y la orientación laboral, la formación, la intermediaciación, el análisis del mercado de trabajo, la identificación y atención a determinados colectivos con dificultades de inserción, la gestión de subvenciones o la I+D+I, entre otras.

En el año 2006 aparece una nueva especialidad de formación para el empleo denominada agente de desarrollo local. Dicha especialidad ya se había impartido previamente a principios de los años noventa, cuando se creó el correspondiente CNO. Curiosamente, los módulos que se imparten en este curso van más allá de las funciones recogidas en las distintas órdenes de subvenciones para la contratación de AEDL. Este hecho, unido a la diferente denominación utilizada (no aparece la palabra empleo), nos hace plantearnos una definición más amplia de la profesión que no se limita únicamente a los AEDL subvencionados. Este programa formativo atribuye a los ADL como objetivo principal dinamizar, analizar y diagnosticar el territorio de intervención y poner en marcha una estrategia de desarrollo económico, social y cultural encaminada a conseguir una mejora de la calidad de vida a nivel local.

Se observa aquí una misión mucho más amplia que la establecida en las funciones vistas hasta ahora. Tampoco se indica en ningún momento que estos profesionales deban trabajar necesariamente por cuenta ajena en una Administración Pública. Por lo tanto, se abre la puerta de la profesión a profesionales autónomos o que desempeñen su trabajo en empresas consultoras, asociaciones empresariales, sindicatos, universidades o entidades sin ánimo de lucro, entre otras.

En cuanto al contenido de este curso, destacan los contenidos relacionados con el desarrollo rural, el asociacionismo y la economía social.

Añadiendo estas funciones ya podemos obtener, sin duda, un perfil profesional mucho más amplio. Este perfil se completará con otras funciones que veremos en apartados posteriores.

\section{LA FORMACIÓN UNIVERSITARIA EN DESARROLLO LOCAL}

Como ocurre con todas las profesiones que requieren una cierta complejidad, en el caso que nos ocupa las universidades tampoco se han quedado al margen, 
aunque en este caso vamos a encontrar una serie de particularidades que no se observan en otros casos. Ello es debido fundamentalmente a dos aspectos: por una parte, el carácter multidisciplinar de la profesión y la inexistencia de una titulación universitaria previa a la aparición de la misma.

Respecto a la primera cuestión, la normativa que regula las subvenciones, únicamente establece como requisito para ejercer la profesión contar con una titulación universitaria de grado, diplomatura o licenciatura. La realidad nos confirma este carácter multidisciplinar de los profesionales. Aunque existe un predominio de titulaciones relacionadas con las ciencias sociales, encontramos un amplio abanico que abarca la práctica totalidad de titulaciones.

La inexistencia de una titulación universitaria con carácter previo a la aparición de una profesión que requiere titulación universitaria y el hecho de que tampoco haya existido esa titulación de grado, licenciatura o diplomatura en los años posteriores, nos sitúa ante una circunstancia bastante insólita que ha dificultado la configuración y el reconocimiento profesional. Este problema no lo han tenido otras profesiones como es el caso de los trabajadores sociales, los educadores sociales o los dietistas y nutricionistas, por citar algunas de las profesiones que recientemente han configurado colegios profesionales.

Sin embargo, algunas universidades apostaron por incluir estos estudios en su oferta formativa de postgrado. En este sentido, algunas universidades ya cuentan con esa oferta formativa de postgrado en desarrollo local desde hace aproximadamente una década. En este hecho también ha influido la existencia de subvenciones autonómicas para la formación para aquellos agentes de empleo y desarrollo local subvencionados.

Coincidiendo con la entrada en vigor de los Máster Oficiales contemplados en el Espacio Europeo de Educación Superior, en el año 2007, más de veinte años después de la aparición de la profesión en España, encontramos por primera vez la existencia de titulaciones universitarias oficiales en desarrollo local. Y son precisamente las universidades valencianas las que toman la iniciativa en esta materia. Por una parte, la Universidad de Alicante pone en marcha el Máster Oficial en Desarrollo Local e Innovación Territorial. Paralelamente, el Instituto Universitario de Desarrollo Local, integrado por las universidades de Valencia y Jaume I de Castelló, ponen en marcha el Máster Oficial en Gestión y Promoción del Desarrollo Local. Sin duda alguna, son dos hechos relevantes que van a ayudar al reconocimiento de la profesión en los próximos años.

\section{CONCLUSIÓN}

A lo largo de los últimos años, la colaboración entre los diferentes Agentes Económicos y las Administraciones Locales ha generado un conjunto amplio de experiencias y buenas prácticas, que han puesto de manifiesto la necesidad de continuar y profundizar en este trabajo conjunto. La cooperación institucio- 
nal entre administraciones públicas y agentes económicos y sociales es un elemento indispensable para la puesta en marcha de medidas eficaces en materia de Políticas Activas de Empleo, como también lo es la dinamización de estas políticas en el ámbito local.

El desarrollo local participativo apuesta por una economía sostenible que siga un patrón de crecimiento que concilie el desarrollo económico, social y ambiental en una economía productiva y competitiva, que favorezca el empleo de calidad, la igualdad de oportunidades y la cohesión social, y que garantice el respeto ambiental y el uso racional de los recursos naturales, de forma que permita satisfacer las necesidades de las generaciones presentes sin comprometer las posibilidades de las generaciones futuras para atender sus propias necesidades.

Del mismo modo, este modelo asume los principios orientadores de la economía social que dan primacía de las personas y del fin social sobre el capital, y los nuevos conceptos que surgen a partir de ella como el emprendimiento social y la responsabilidad social corporativa, principios que persiguen la promoción de la solidaridad interna y con la sociedad que favorezca el compromiso con el desarrollo local, la cohesión social y la sostenibilidad.

La creación de un Colegio Profesional de Técnicos en gestión del desarrollo local se presenta como un elemento articulador esencial para avanzar desde la base hacia un modelo socioeconómico basado en el desarrollo local participativo. 